\title{
Demographic and Behavioural Factors Associated with Uptake of at least Three Doses of Intermitted Preventive Treatment of Malaria in Pregnancy (IPTp-SP3) among Nigerian Women
}

\author{
Article by Okoh, O.M ${ }^{1}$, Babalola, $\mathrm{S}^{1,2}$, McCartney-Melstad, $\mathrm{A}^{1}$, Tweedie, $\mathrm{I}^{1}$, Amosu, A. $\mathrm{M}^{3}$ \\ ${ }^{1}$ Health Communication Capacity Collaborative Project and Johns Hopkins University \\ Center for Communications Program \\ ${ }^{2}$ Johns Hopkins University School of public health \\ ${ }^{3}$ Babcock University School of public and allied health \\ E-mail: mathewokoh@yahoo.com ${ }^{1}$
}

\begin{abstract}
Malaria is a vector borne protozoa disease that is endemic in Nigeria and continues to present significant public health challenge to the Nigerian government. Pregnant women are especially vulnerable to malaria infection due to their compromised immunity. Malaria contributes significantly to poor pregnancy outcomes like low birth weight, premature delivery, still birth, spontaneous abortion and neonatal death.

The use of IPTp-SP has been proven to be a safe, cheap and effective means to prevent malaria and its consequences among pregnant women. Consequently, pregnant women are expected to receive a minimum of three doses of IPTp-SP beginning from the $13^{\text {th }}$ week of pregnancy. In spite of this, uptake of IPTp-SP by pregnant women still remains low in Nigeria.

This research investigated the demographic and behavioural factors that were associated with uptake of at least three doses of IPTp-SP among women who had a live birth in the two years preceding the survey. The study was quantitative in design and used cross sectional data that included 3205 women from Akwa Ibom, Kebbi and Nasarawa states.

Findings from this study suggest that ANC attendance is the most important factor associated with uptake of a minimum of three doses of IPTp-SP (IPTp-SP3). This might not be unconnected to the IPTp-SP strategy being largely a facility based intervention. Household wealth index exhibited a marginally significant relation with uptake of IPTp-SP3. After adjusting for cluster level effects and other potential confounders, a woman's decision making autonomy and state where she was resident were found to be significantly associated with uptake of IPTp-SP3. An unexpected but logical finding was the inverse relationship between regular use of bed net and uptake of IPTp-SP3. This finding has implication for how bed net use is presented to pregnant women.

Results from this study suggests that, improved access to ANC services, improved women's autonomy, and educating pregnant women that regular bed net use does not preclude the need for IPTp-SP utilization could go a long way in improving uptake of IPTp-SP among pregnant women in Nigeria.
\end{abstract}

Keywords: Malaria, Pregnancy, Ante natal care, IPTP-SP, Behaviour, Nigeria.

\section{Introduction}

Malaria is a protozoa disease that results from the bite of infected female anopheles mosquitoes and affects all age groups. However, pregnant women are one of the most vulnerable groups to malaria due to their low level of immunity. Malaria in pregnancy accounts for $11 \%$ of maternal mortality in Nigeria (NMEP, NPoPC, NBS, \& ICF, 2016). In addition, malaria in pregnancy is associated with several poor outcomes for both the pregnant woman and her unborn child. Intrauterine growth retardation, intra uterine death, still birth, premature delivery and low birth weight are some of the documented consequences of malaria among pregnant women (WHO 2017, Marchant et al., 2004; Verhoeff et al., 1998). Maternal anaemia, postpartum morbidity (WHO 2017, Shulman et al., 1996), and spontaneous abortions (McGready, White, \& Nosten, (2011); McGready et al., 2012) have all been associated with malaria in pregnant women. 
In areas where malaria is endemic including Nigeria, infection in pregnant women is often asymptomatic due to partial immunity from repeated exposure. Infection may therefore go unsuspected, but is often associated with heavy placental parasitamia, with its consequent poor pregnancy outcomes (Steketee, Wirima, \& Campbell 1996). In order to mitigate the deleterious effect of asymptomatic malaria infection in pregnant women, the World Health Organization recommends that pregnant women in malaria endemic areas receive at least three doses of intermittent preventive treatment (IPTp) with sulphadoxine-pyrimethamine (SP) in addition to the regular use of insecticidetreated bed nets (WHO, 2014). In early 2014, Nigeria adopted the three dose policy for IPTp-SP implementation as recommended by the WHO (FMOH 2014).

In spite of the demonstrated efficacy, safety, affordability and ease of IPTp-SP implementation in Nigeria and other parts of the world (WHO 2014; Aziken, Akubuo, \& Gharoro, 2011; Mboye, Bygbjerg, \& Magnussen, 2008; Peter, 2013; Takem, Achidi, \& Ndumbe, 2009), IPTp-SP uptake remains very low in Nigeria (NMEP, NPoPC, NBS, \& ICF, 2016). Furthermore, there has been no study to examine the factors associated with uptake of three doses of IPTp-SP (IPTp-SP3) among pregnant women in Nigeria. This study therefore, aimed to fill this gap by highlighting the demographic and behavioural factors associated with uptake of three doses of IPTp-SP among pregnant women in Nigeria.

\section{Objectives}

The main objective of this study is to identify the factors that are associated with the uptake of IPTp-SP3 among pregnant women in Nigeria. Identifying these factors will contribute to the existing body of knowledge in the area of IPTp-SP uptake and could be useful in improving IPTp-SP intervention among pregnant women in Nigeria.

\section{The specific objectives are}

- To identify the demographic characteristics associated with the uptake IPTp-SP3 among pregnant women in Nigeria; and

- To examine the influence of ANC attendance on uptake of IPTp-SP3 among pregnant women in Nigeria

\section{Materials and methods}

\section{Design}

This analysis was based on a cross sectional household survey data, collected as baseline for a project funded by the United States Agency for International Development (USAID). The project was designed to promote adoption of appropriate malaria behaviors and thereby decrease malaria disease burden in Nigeria through social and behaviour change communication strategies.

\section{Primary data collection plan}

Multistage cluster sampling design was used to select a representative sample of households with at least one child under the age of five years in three survey states (Akwa Ibom, Kebbi and Nasarawa). The sampling process involved selecting the local government area, the enumeration area (cluster), and then the household. Sample size for the study was based on proportion of the population with positive attitudes towards bed nets. Since such information was not available for any of the study states, it was assumed that this indicator was $50 \%$. This level of prevalence of positive attitudes is ideal for estimating the required sample size as it provides maximum variability. In addition, it was also assumed that program activities will result in an increase of 10 percentage points in the prevalence of positive attitudes towards bed nets. Based on these statistics and a power of 0.90 , a sample size of 1200 households per state was achieved after accounting for a $10 \%$ non-response rate

\section{Data collection}

Data was collected between July and October 2015. In each selected household, the mother of a child aged less than five years was invited to complete an interviewer-administered semi-structured questionnaire. One woman was randomly selected in households where there was more than one 
eligible woman. However, only women who had a live birth in the two years preceding the survey and currently in union were included in this analysis.

\section{Data analysis}

Multilevel modeling was used to assess the factors associated with obtaining at least three doses of SP (IPTp-SP3) during the pregnancy of the most recent live birth in the last two years (dependent variable). Multilevel analysis technique was chosen to account for the hierarchical nature of the data (individuals nested within clusters).

The dependent variable was categorized as "Yes" if the woman received at least three doses of SP and "No" if she received less than three doses. The independent variables examined included: (i) demographic variables (ii) ANC attendance (iii) regular use of bed net and (iv) a woman's participation in decisions concerning her own health (described as autonomy). The effect of urban residence, state of residence, proportion of women with less than a secondary education in a community and community level poverty index on uptake of three doses of IPTp-SP were also examined. Community level educational and poverty status were generated by computing the nonself-mean.

\section{Multilevel models specification}

Three models with variables of interest were fitted using the melogit command in Stata 14. The first model (intercept only/empty model) was used to check if the data has justifiable evidence to assess random effect at the cluster level. The second model was used to examine individual level factors, to assess their relationship with IPTp-SP3 uptake. The third model simultaneously examine the effect of both individual and community level factors. All analysis were interpreted at $\mathrm{p}<0.05$

\section{Ethical consideration}

Ethical approval for data collection was obtained from Johns Hopkins School of public health with IRB \#6234 as well as from the National Health Research Ethics Committee (NHREC) through the Federal Ministry of Health of Nigeria with approval number NHREC/01/01/2007-10/06/2015.

\section{Results}

Three thousand two hundred and five women who were in union reported that they had a child in the last two years and were included in the analysis. Demographic characteristics of the respondents are shown in table 1 . The mean age of these respondents was 28 years $(\mathrm{SD}=7)$. A little over half (55.1) of them reported to have had formal education. Less than half of the respondents $(49.1 \%)$ were of the Christian faith, while $48.5 \%$ reported to be Muslim. Three-quarters (77\%) of all respondents reported to be resident in a rural area.

Table 1. Percentage distribution of respondents' socio-demographic characteristics ( $\mathrm{n}=3205)$

\begin{tabular}{|l|l|}
\hline Socio-demographic Characteristics & $\mathbf{N}(\boldsymbol{\%})$ \\
\hline Age group & \\
$15-24$ & $974(30.4)$ \\
$25-34$ & $1609(50.2)$ \\
$35-44$ & $520(16.2)$ \\
$45+$ & $102(3.2)$ \\
\hline Religion & \\
Christian & $1575(49.1)$ \\
Muslim & $1553(48.5)$ \\
Other & $77(2.4)$ \\
\hline Parity & \\
$1-2$ & $1253(39.1)$ \\
$3-4$ & $1143(35.7)$ \\
$5+$ & $809(25.2)$ \\
\hline Education level & \\
\hline
\end{tabular}


DOI: 10.21522/TIJPH.2013.06.01.Art009

ISSN: $2520-3134$

\begin{tabular}{|l|l|}
\hline None & $1439(44.9)$ \\
Primary & $787(24.6)$ \\
Secondary & $844(26.3)$ \\
Tertiary & $135(4.2)$ \\
\hline Type of place of residence & \\
Rural & $2470(77.1 \%)$ \\
Urban & $735(23.9 \%)$ \\
\hline Wealth index & $669(20.9)$ \\
Poorest & $642(20.0)$ \\
Second & $653(20.4)$ \\
Middle & $626(19.5)$ \\
Fourth & $615(19.2)$ \\
Richest & \\
\hline State of residence & $961(30.0)$ \\
Akwa Ibom & $1070(33.4)$ \\
Kebbi & $1174(36.6)$ \\
Nasarawa & \\
\hline
\end{tabular}

\section{ANC attendance and use of malaria preventive measures}

The proportion of women who reported attending ANC clinic as well as use of malaria preventive measures during pregnancy is shown in Table 2. Two-thirds (67.5\%) of the respondents attended ANC at least once during their last pregnancy, and half (50.3\%) reported doing so at least four times. Majority (85.7\%) went to a government health facility for their ANC services. Less than one fifth of the women reported to have received a minimum of three doses of IPT-SP as recommended. Similarly, only $37.3 \%$ of the respondents reported sleeping inside a bed net every night as recommended.

Table 2. Utilization of pregnancy related health care services

\begin{tabular}{|l|l|}
\hline & $\mathbf{n}(\boldsymbol{\%})$ \\
\hline Uptake of health care services & \\
\hline Did not attend any ANC & $1041(32.5)$ \\
Attended ANC less than 4 times & $550(17.2)$ \\
Attended ANC at least 4 times & $1613(50.3)$ \\
\hline Use of bed net & $584(18.32$ \\
Never used bed net & $1423(44.4)$ \\
Use bed net but not every night & $1198(37.4)$ \\
Use bed net every night & $2219(69.2)$ \\
\hline $\begin{array}{l}\text { Uptake of IPTp-SP } \\
\text { Did not take IPTp-SP } \\
\text { Took less than 3 doses of IPTp-SP }\end{array}$ & $389(12.1)$ \\
Took at least 3 doses of IPTp-SP & $597(18.6)$ \\
\hline
\end{tabular}

\section{Variation in IPTp-SP3 uptake with behavioural and socio-demographic characteristics}

Uptake of IPTp-SP3 was directly related to ANC attendance but inversely associated with use of bed net. Women who reported attending ANC a minimum of four times were more likely (27.5\%) to report uptake of IPTp-SP3 compared to women who reported not attending ANC at all or attended ANC less than four times $(9.6 \%) \mathrm{X}^{2}=169.7 ; \mathrm{p}<0.001$. The proportion of women who reported uptake of IPTp-SP3 was lower (14.9\%) among women who sleep inside a bed net every night compared to other women $(20.9 \%) \mathrm{X}^{2}=17.9 ; \mathrm{p}<0.001$.

Uptake of IPTp-SP3 did not significantly differ by parity, but showed significant variation by age, religion, education, wealth status and place of residence (table 3). Women in the age group 25-34 
years $(20.5 \%)$ and $35-44$ years $(21.0 \%)$ were more likely to report uptake of IPTp-SP3 compared to women in other age groups $(\mathrm{p}=0.001)$. Prevalence of IPTp-SP3 uptake was highest $(27.4 \%)$ among women with tertiary education and lowest (15.5\%) among those who reported not having a formal education $(\mathrm{p}<0.001)$. Similarly, prevalence of IPTp-SP3 uptake was highest $(23.7 \%)$ among those in the highest wealth category and lowest $(12.6 \%)$ among those in the lowest wealth category $(\mathrm{p}<0.001)$.

Table 3. Relationship between IPTp-SP3 uptake and respondents' socio-demographic profile

\begin{tabular}{|l|l|l|}
\hline Socio-demographic Characteristics & $\begin{array}{l}\text { Proportion of women } \\
\text { who took IPTp-SP3 }\end{array}$ & $\mathbf{X}^{2}$ (p value) \\
\hline Age group & 15.0 & \\
$15-24$ & 20.5 & \\
$25-34$ & 21.0. & \\
$35-44$ & 11.8 & $17.3(0.001)$ \\
$45+$ & & \\
\hline Religion & 19.7 & \\
Christian & 18.8 & $11.4(0.003)$ \\
Muslim & 3.9 & \\
Other & & \\
\hline Parity & 17.1 & $4.7(0.094)$ \\
$1-2$ & 18.7 & \\
3-4 & 20.9 & \\
$5+$ & & \\
\hline Education level & 15.5 & \\
None & 22.5 & \\
Primary & 19.0 & \\
Secondary & 27.4 & \\
Tertiary & & \\
\hline Type of place of residence & 16.2 & \\
Rural & 26.7 & \\
Urban & 12.6 & \\
\hline Wealth index & 19.0 & \\
Poorest & 18.4 & \\
Second & 20.0 & \\
Middle & 23.7 & \\
Fourth & 15.0 & \\
Richest & 15.1 & \\
\hline State of residence & 24.9 & \\
Akwa Ibom & & \\
Kebbi & & \\
Nasarawa & & \\
\hline & & \\
\hline
\end{tabular}

\section{Individual and contextual factors associated with uptake of IPTp-SP3}

Table 4 presents the result of multilevel modeling of factors that are independently associated with uptake of IPTp-SP3. The empty model (model 1) indicated that there was significant variability in the odds of IPTp-SP3 uptake across communities $\left(\sigma^{2}=.75, \mathrm{p}<0.001\right)$. The intra-cluster correlation also indicated that $18.6 \%$ of the variation in the outcome variable was due to unexplained contextual factors.

In model 2, we adjusted for individual level variables only, to examine their relative association with uptake of IPTp-SP3. Individual sociodemographic, ANC attendance and regular use of bed net were simultaneously adjusted for in this model. Religion, use of bed net, ANC attendance and woman's autonomy were statistically significantly associated with uptake of IPTp-SP3. The variance in IPTp-SP3 uptake remained significant in this model as well $\left(\sigma^{2}=0.59 ; \mathrm{p}<0.001\right)$ 
DOI: 10.21522/TIJPH.2013.06.01.Art009

ISSN: $2520-3134$

After adjusting for individual and community level factors in model 3, Religion, household wealth status, use of bed net, ANC attendance, woman's autonomy and state of residence were found to be significantly associated with uptake of IPTp-SP3. Age, marital status, parity, community poverty level did not seem to have a significant association with uptake of IPTp-SP3. The measures of variation from this model indicated significant variability across communities in the outcome variable $\left(\sigma^{2}\right.$ $=0.45, \mathrm{p}<0.001)$. The variation in the outcome variable due to unmeasured cluster level factors was reduced to $12.0 \%$ in this model. However this was still statistically significant.

Table 4. Multilevel analysis of factors independently associated with uptake of IPTp-SP3

\begin{tabular}{|c|c|c|c|}
\hline Respondents characteristics & $\begin{array}{l}\text { Model } 1 \\
\text { Empty } \\
\text { model } \\
\end{array}$ & $\begin{array}{l}\text { Model } 3 \\
\text { Individual } \\
\text { variables } \\
\end{array}$ & $\begin{array}{l}\text { Model } 4 \\
\text { Community } \\
\text { \& individual } \\
\text { variables } \\
\end{array}$ \\
\hline Fixed effects & & OR $(95 \% \mathrm{CI})$ & OR $(95 \% \mathrm{CI})$ \\
\hline $\begin{array}{l}\text { Age group } \\
15-24 \text { (ref) } \\
25-34 \\
35-44 \\
45+ \\
\end{array}$ & & $\begin{array}{l}1.00 \\
1.24(.96-1.60) \\
1.20(.86-1.69) \\
.63(.31-1.25) \\
\end{array}$ & $\begin{array}{l}1.00 \\
1.26(.98-1.62) \\
1.22(.87-1.72) \\
.66(.33-1.32) \\
\end{array}$ \\
\hline $\begin{array}{l}\text { Religion } \\
\text { Christian (ref) } \\
\text { Muslim } \\
\text { Other } \\
\end{array}$ & & $\begin{array}{l}1.00 \\
1.36(1.01-1.82)^{*} \\
.33(.09-1.29)\end{array}$ & $\begin{array}{l}100 \\
.92(.65-1.31) \\
.25(.06-.95)^{*}\end{array}$ \\
\hline $\begin{array}{l}\text { Parity } \\
1-2 \text { (ref) } \\
3-4 \\
5+ \\
\end{array}$ & & $\begin{array}{l}1.00 \\
.99(.78-1.26) \\
1.13(.85-1.50) \\
\end{array}$ & $\begin{array}{l}1.00 \\
.98(.77-1.25) \\
1.10(.83-1.46)\end{array}$ \\
\hline $\begin{array}{l}\text { Education level } \\
\text { None (ref) } \\
\text { Primary } \\
\text { Secondary } \\
\text { Tertiary } \\
\end{array}$ & & $\begin{array}{l}1.00 \\
1.23(.92-1.63) \\
.97(.71-1.33) \\
1.32(.78-2.24) \\
\end{array}$ & $\begin{array}{l}1.00 \\
1.32(.99-1.77) \\
1.07(.78-1.46) \\
1.37(.81-2.31)\end{array}$ \\
\hline $\begin{array}{l}\text { Wealth index } \\
\text { Poorest (ref) } \\
\text { Second } \\
\text { Middle } \\
\text { Fourth } \\
\text { Richest }\end{array}$ & & $\begin{array}{l}1.00 \\
1.21(.85-1.72) \\
1.09(.75-1.58) \\
1.24(.83-1.84) \\
1.26(.82-1.93) \\
\end{array}$ & $\begin{array}{l}1.00 \\
1.27(.89-1.81) \\
1.21(.83-1.78) \\
1.53(1.00- \\
2.32) \ddagger \\
1.59(1.00- \\
2.52) \ddagger\end{array}$ \\
\hline $\begin{array}{l}\text { Use of bed net } \\
\text { Sleep inside bed net every } \\
\text { night } \\
\text { Does not use bed net every } \\
\text { night }\end{array}$ & & $\begin{array}{l}.70(.56-.89)^{* *} \\
1.00\end{array}$ & $\begin{array}{l}.70(.56-.88)^{* *} \\
1.00\end{array}$ \\
\hline $\begin{array}{l}\text { ANC attendance } \\
\text { Attended ANC less than } 4 \\
\text { times } \\
\text { Attended ANC at least } 4 \text { times }\end{array}$ & & $\begin{array}{l}1.00 \\
3.53(2.80- \\
4.44)^{* * *} \\
\end{array}$ & $\begin{array}{l}1.00 \\
3.47(2.75- \\
4.38)^{* * *} \\
\end{array}$ \\
\hline $\begin{array}{l}\text { Decision over own health } \\
\text { Self alone (ref) } \\
\text { Husband alone } \\
\text { Jointly with husband } \\
\text { Others }\end{array}$ & & $\begin{array}{l}1.00 \\
.65(.49-85)^{* *} \\
.70(.53-.91)^{* *} \\
.68(.34-1.36) \\
\end{array}$ & $\begin{array}{l}1.00 \\
.63(.48-.83)^{* *} \\
.65(.50-.86)^{* *} \\
.74(.37-1.49) \\
\end{array}$ \\
\hline
\end{tabular}




\begin{tabular}{|c|c|c|c|}
\hline $\begin{array}{l}\text { Type of place of residence } \\
\text { Rural } \\
\text { Urban (ref) }\end{array}$ & & & $\begin{array}{l}.76(.56-1.04) \\
1.00\end{array}$ \\
\hline $\begin{array}{l}\text { State of residence } \\
\text { Akwa Ibom (ref) } \\
\text { Kebbi } \\
\text { Nasarawa }\end{array}$ & & & $\begin{array}{l}1.00 \\
3.00(1.56- \\
5.76)^{* *} \\
2.39(1.50- \\
3.81)^{* * *} \\
\end{array}$ \\
\hline $\begin{array}{l}\text { Community wealth status } \\
\text { Low (ref) } \\
\text { Medium } \\
\text { High } \\
\end{array}$ & & & $\begin{array}{l}1.00 \\
1.37(.93-2.03) \\
1.62(.95-2.77)\end{array}$ \\
\hline $\begin{array}{l}\text { Community education } \\
\text { Low (ref) } \\
\text { Medium } \\
\text { High } \\
\end{array}$ & & & $\begin{array}{l}1.00 \\
.83(.56-1.24) \\
.64(.37-1.10) \\
\end{array}$ \\
\hline \multicolumn{4}{|l|}{ Random effects } \\
\hline Community variance (SE) & $\begin{array}{l}0.75 \\
(0.15) * * *\end{array}$ & $0.59(0.13) * * *$ & $0.45(0.11)^{* * *}$ \\
\hline $\operatorname{ICC}(\%)$ & 18.6 & 15.2 & 12.0 \\
\hline \multicolumn{4}{|l|}{ Model fit statistics } \\
\hline Log-likelihood & -1482.35 & -1388.99 & -1374.71 \\
\hline AIC & 2968.70 & 2819.99 & 2805.42 \\
\hline
\end{tabular}

\section{Discussion}

Findings from this study indicated that uptake of any dose of IPTp-SP was low just as has been documented by other studies in Nigeria and across Sub-Saharan Africa (Brieger, 2012; Akinleye, Falade, \& Ajayi, 2009; Nduka, Nwosu, \& Oguariri, 2011; NMEP, NPoPC, NBS, \& ICF, 2016; Hughes, 2011). Uptake of the recommended minimum of three doses of IPTp-SP by this study population was less than one fifth as was also reported by the most recent malaria indicator survey conducted in Nigeria (NMEP, NPoPC, NBS, \& ICF, 2016). These findings suggest a significant failure to meet the country's target of reaching all pregnant women with IPTp-SP as contained in its national guideline for the prevention and control of malaria in pregnant women (FMOH, 2014).

Women who attended at least four ANC clinic visits were more than three times as likely to report uptake of IPTp-SP3 compared to women who attended ANC less than four times. This is not at all surprising, giving the fact that, IPTp-SP intervention is a facility based intervention designed to provide SP to pregnant women during ANC visits. Therefore, the more ANC visits a woman makes during pregnancy, the more chances she gets to receive IPTp-SP. This finding is in consonance with that of other researchers across Sub-Saharan Africa who have documented positive relationship between the numbers of ANC visits with IPTp-SP uptake. Mutulei (2013), for example, reported that Kenyan women who initiate ANC visits in the second month of pregnancy were more than ten times as likely to report uptake of at least two doses of IPTp-SP compared to women who started ANC in the sixth month of their pregnancy. Sikambale et al (2013) also reported a significant relationship between uptake of IPTp-SP3 and the gestational age at which women attended ANC for the first time as well as the number of times women attended ANC in Zambia. In Tanzania, one facility based and two population based studies all concluded that women who initiate ANC attendance in the first trimester of pregnancy were significantly more likely to receive IPTp-SP2 compared to women who initiated ANC attendance later in pregnancy (Nganda, Drakeley, Reyburn, \& Marchant, 2004; Exavery et al 2014; Kibusi, Kimunai, \& Hines, 2015). The possible explanation here is that when pregnant women initiate ANC visits early on in their pregnancy, they have a longer window period within which to make subsequent ANC visits and thereby increasing their likelihood of receiving 
IPTp-SP. However, Onoka, Hanson \& Onwujekwe (2012) in a 2010 review of IPTp-SP coverage in Enugu state, South Eastern Nigeria noted missed opportunities to provide IPTp-SP to pregnant women. They observed that, most pregnant women who were due to receive IPTp-SP as recommended by WHO during their ANC attendance, did not receive IPTp-SP (Onoka, Hanson \& Onwujekwe (2012). They therefore concluded that the number of ANC visits alone is not enough to guarantee adequate uptake of IPTp-SP. This position was further buttressed by Exavery and colleagues in 2014 when they noted that the quality of service provided during ANC visit might be more important than just the number of visits with respect to IPTp-SP uptake (Exavery et al, 2014). Uptake of IPTp being largely a facility based intervention, is dependent on the availability of sulfadoxine-Pyrimethamine in the facility and the provider offering it to the woman. The fact remains however, that a woman's opportunity to receive IPTp-SP increases if she attends ANC regularly. Evidence from Gabon and Ghana have shown that women are more likely to receive the recommended number of IPTp-SP doses if they attend a minimum of four ANC visits (BouyouAkotet, Mawili-Mboumba, \& Kombila, M. (2013); Owusu-Boateng, \& Anto, (2017).

In this study, religion, woman's autonomy and household wealth status were statistically significantly associated with uptake of IPTp-SP3. Women who did not identify themselves as Christian or Muslims were significantly less than likely to have reported uptake of IPTp-SP3 compared to other women. Women from the households in the highest two quintiles were more likely to receive IPTp-SP3 compared to those in the poorest households. Marital status, education, age, and parity did not show a significant relationship with IPTp-SP3 uptake. This finding is consistent with studies from other parts of Nigeria. For example, studies conducted in Shagamu and Ibadan, South West Nigeria did not show a significant relationship between age, education, parity and IPTp-SP uptake (Amoran, Ariba, \& Iyaniwura, 2012; Olukemi, Emmanuel, \& Olusina, 2009). Although we examined uptake of IPTp-SP3, these previous studies investigated IPTp-SP2. We however, identified two studies that specifically examined uptake of IPTp-SP3. While one of the studies did not adjust for the confounding effects of other variables (Sikambale, Halwindi, \& Baboo, 2013), the other study did not adjust for demographic factors in the model specified (Owusu-Boateng, \& Anto, 2017).

Unlike ANC attendance, women who reported sleeping inside a bed net every night were significantly less likely to report receiving IPTp-SP3 compared to those who either did not use bed net at all or used a bed net infrequently. This finding is at variance with the findings of a meta-analysis conducted by Hill et al (2013) which determined that women who reported sleeping inside a bed net were three and half times likely to report uptake of a minimum of two doses of IPTp-SP. It should be noted however, that this present study categorized women as users of bed net only if they reported sleeping inside a bed net every night compared to these previous studies that categorized women as bed net users if they reported sleeping inside a bed net the night before the survey. It is likely that women who sleep inside a bed net every night, might feel that they are adequately protected from mosquito bites, and as such do not see the need to swallow medications especially for an illness they do not have. In a study conducted in Tanzania, more than 9 in 10 women interviewed felt that use of bed nets was a good measure to prevent malaria during pregnancy but less than half of them felt the same way for IPTp-SP use (Nganda, Drakeley, Reyburn, \& Marchant 2004). Similarly, Olukemi and her colleagues in 2009 documented that more than half of the women surveyed who gave a reason for not using IPTp-SP said they did not use IPTp-SP because they preferred other preventive measures (Olukemi, Emmanuel, and Olusina, 2009).

While regular use of bed net can contribute to reducing poor birth outcomes like maternal anemia, placental infection and low birth weight attributable to malaria infection (WHO, \& UNICEF, 2003). It should be noted however, that the protective effect of bed net only accrues when the pregnant woman is lying down inside it. Pregnant women can still get bitten by mosquito as they go about their daily chores, thereby exposing them to infection with the malaria parasite. Available evidence indicates that, the Anopheles mosquito responsible for transmitting malaria is gradually modifying its feeding behaviour from late evenings when people are sleeping to early evenings when people are still active (Russell, Beebe, Cooper, Lobo, \& Burkot, 2013; Sokhna, Ndiath, \& Rogier, 2013; Thomsen et al 2017). Nganda and colleagues observed that maternal anemia was least prevalent among women who use IPTp-SP in addition to sleeping inside a bed net when compared to those who use bed net alone or 
IPTp-SP alone (Nganda, Drakeley, Reyburn, \& Marchant 2004). Similarly, Fokam and his colleagues in a hospital based cross-sectional study among pregnant women attending ANC, reported the prevalence of malaria to be lowest among those using both IPTp-SP and bed net compared to those using either IPTp-SP or bed net only (Fokam, Ngimuh, Anchang-Kimbi, \& Wanji, 2016). Pregnant women should therefore be encouraged to use multiple protective measures against malaria for improved outcomes.

Available literature describes autonomy to include the ability of individuals to make decisions without recourse to other people (Nayak \& Varambally, 2017; Wypij, \& Gupta, (2001). In this study we measured autonomy by a woman's decision making status. Findings from the current study indicated that women whose health decisions are made jointly with husband or by husband alone were significantly less likely to report uptake of IPTp-SP3 compared to women who make their own health decisions themselves. Researchers from Nigeria, Ethiopia, Eritrea, India and Nepal have all shown that women with higher autonomy are more likely to use maternal and preventive health care services (Nayak \& Varambally, 2017; Adhikar 2016; Mahapatro, 2012; Mistry, Galal, \& Lu, 2009; Ononokpono, \& Odimegwu 2014; Woldemicael, 2007). This universality in agreement on the role of women's autonomy in health care behaviour indicates that this is an important area for intervention in the bid to improve uptake of maternal health care services. There is therefore the need for interventions aimed at improving uptake of maternal health care services to address those social, cultural and other factors that undermine women's decision-making autonomy.

\section{Study limitations and strengths}

The results from this study should be interpreted bearing in mind that it is not without limitations. Firstly, the cross sectional nature of this study does not allow for causal inferences to be made. The fact that women were asked to recall services received in the past two years exposes the findings to recall bias. However, this is the standard period for measuring uptake of IPTp-SP internationally. Furthermore, our study population included a few women who had a live birth just before implementation of the three dose policy began. A situation that could have influence the number of IPTp-SP doses they received. The non-inclusion of some potential confounders (e.g., access to health services, availability of supplies, etc.) in the analyses due to data limitation may have biased the estimates of measured variables. Finally, individual wealth status was estimated based on ownership of some household assets. This is an indirect measure of wealth status due to non-availability of information on personal income. However, this method of estimating wealth status is widely used and has been shown to be reliable (Filmer, \&Pritchett, 2001).

Despite these limitations, this study has several strengths. Firstly, this is the largest population based study in the area of IPTp-SP in Nigeria with over 3000 respondents. Secondly, this is the first study to examine factors associated with uptake of three doses of IPTp-SP in Nigeria. Thirdly, the data is representative of the three states included in the study. This is the first study in Nigeria to employ multilevel modeling in this area of research thereby allowing for the examination of the clustering effect of the outcome variable. Finally, this study identifies factors that could be targeted for interventions and policy initiatives.

\section{Conclusions and recommendations}

This study further confirms the low uptake of IPTp-SP3 as recommended among women in Akwa Ibom, Kebbi and Nasarawa states of Nigeria. There is therefore a need for interventions targeted at those behavioural factors identified by this study to be associated with uptake of IPTp-SP3. This should be in addition to ensuring adequate supply of sulfadoxine-pyrimethamine and appropriate provider practices with respect to IPTp-SP administration to pregnant women.

This study is the first to show the relationship between regular use of bed net, women's autonomy and uptake of IPTp-SP3. This finding has both policy and programme implication for the way use of bed net intervention is presented to pregnant women in Nigeria. Health care workers should continue to encourage pregnant women to use IPTp-SP as recommended in addition to consistent use of bed net. 
- Finally, results from this investigation demonstrates that ANC services continues to be an effective medium for the implementation of the IPTp-SP intervention for pregnant women in Nigeria. Consequently, early and adequate ANC attendance should be vigorously promoted among pregnant women.

\section{Acknowledgements}

This analysis was based on data collected with support of the US President's Malaria Initiative. The contents of this report are the sole responsibility of the authors and does not necessarily represent the views or positions of USAID, PMI, the U.S. Government, or The Johns Hopkins University.

\section{References}

[1].Adhikar, R. (2016). Effect of Women's autonomy on maternal health service utilization in Nepal: a cross sectional study. BMC Women's Health 16:26. DOI 10.1186/s12905-016-0305-7. Available from https://www.ncbi.nlm.nih.gov/pmc/articles/PMC4867085/. Accessed June 262017

[2].Akinleye SO, Falade CO, Ajayi IO. (2009). Knowledge and utilization of intermittent preventive treatment for malaria among pregnant women attending antenatal clinics in primary health care centers in rural southwest, Nigeria: a cross-sectional study. BMC Pregnancy Childbirth, 9:28.

[3].Amoran, O.E., Ariba, A.A., \& Iyaniwura, C.A. (2012). Determinants of intermittent preventive treatment of malaria during pregnancy (IPTp) utilization in a rural town in Western Nigeria. Reproductive Health, 9:12. Available from http://www.reproductive-health-journal.com/content/9/1/12 Accessed June 112015

[4].Aziken, M.E., Akubuo, K.K., \& Gharoro E.P. (2011). Efficacy of intermittent preventive treatment with sulfadoxine-pyrimethamine on placental parasitemia in pregnant women in Midwestern Nigeria. Int J of Gyne obs, 112 (1) 30-33

[5].Bloom, S.S., Wypij, D., Gupta, M.D. (2001). Dimensions of Women's Autonomy and the Influence on Maternal Health Care Utilization in a North Indian City. Demography, 38(1), 67-78.

[6].Bouyou-Akotet, M., K., Mawili-Mboumba, D., P., \& Kombila, M. (2013). Antenatal care visit attendance, intermittent preventive treatment and bed net use during pregnancy in Gabon. BMC Pregnancy and Childbirth, 13:52 Available from http://www.biomedcentral.com/1471-2393/13/52. Accessed November 282017

[7].Brieger W. (2012). Control of malaria in pregnancy: an elusive target. Afr Health. 34:15-18

[8].Exavery, A., Mbaruku, G., Mbuyita, S., Makemba, A., Kinyonge, I.P., \& Kweka, H. (2014). Factors affecting uptake of optimal doses of sulphadoxine-pyrimethamine for intermittent preventive treatment of malaria in pregnancy in six districts of Tanzania. Malaria Journal, 13:22. Available from http://www.malariajournal.com/content/13/1/22. Assessed December 272015.

[9]. Federal Ministry of Health. (2014). National Guidelines and Strategies for Malaria Prevention and Control during Pregnancy. Second Edition Federal Ministry of Health, Nigeria.

[10]. Filmer, D. and Pritchett, L.H. (2001). Estimating wealth effects without expenditure data or tears: an application to educational enrollment in states of India. Demography 38, 115-132.

[11]. Fokam, E.B., Ngimuh, L., Anchang-Kimbi, J.K., \& Wanji, S. (2016). Assessment of the usage and effectiveness of intermittent preventive treatment and insecticide-treated nets on the indicators of malaria among pregnant women attending antenatal care in the Buea Health District, Cameroon. Malar J 15:172 DOI 10.1186/s12936-016-1228-3. Available from https://malariajournal.biomedcentral.com/articles/10.1186/s12936016-1228-3. Accessed July 12017

[12]. Hill, J., Hoyt, J., van Eijk, A.M., D’Mello-Guyett, L., ter Kuile, F.O., Stekette, R., Smith, H., \& Webster, J. (2013). Factors Affecting the Delivery, Access, and Use of Interventions to Prevent Malaria in Pregnancy in Sub-Saharan Africa: A Systematic Review and Meta-Analysis. Plos ONE 10(7) e1001488. https://doi.org/10.1371/journal.pmed.1001488. Available from http://journals.plos.org/plosmedicine/article?id=10.1371/journal.pmed.1001488. Accessed June 262017

[13]. Hughes, A. (2011). Methods to protect pregnant women from malaria are still under-utilized in SubSaharan Africa. Medical News Today. Available from http://www.medicalnewstoday.com/printerfriendlynews.php?newsid. Accessed 20 Nov 2016.

[14]. Kibusi, S.M., Kimunai, E., \& Hines, C.S. (2015). Predictors for uptake of intermittent preventive treatment of malaria in pregnancy (IPTp) in Tanzania. BMC Public Health 15:540 DOI 10.1186/s12889-015-1905-0 
[15]. Mahapatro, SR. (2012). Utilization of Maternal and Child Health Care Services in India: Does women's autonomy matter? The Journal of Family Welfare Vol. 58, No.1 22-33.

[16]. Mistry, R., Galal, O., Lu, M. (2009). "Women's autonomy and pregnancy care in rural India: A contextual analysis" Social Science \& Medicine 69; 926-933.

[17]. Marchant, T., Armstrong-Schellenberg, J., Nathan, R., Abdulla, S., Mukasa, O., Mshinda, H., \& Lengeler, C. (2004). Anaemia in pregnancy and infant mortality in Tanzania.Trop Med Int Health, 9 (2):262-266

[18]. Mbonye, A.K., Bygbjerg, I.B., Magnussen, P. (2008). Intermittent preventive treatment of malaria in pregnancy: A community based delivery-system and its effects on parasitaemia, anaemia and low birth weight in Uganda. Int J Infect Dis, 2:22-29.

[19]. McGready, R., Lee, S.J., Wiladphaingern, J., Ashley, E.A., Rijken, M.J., Boel, M., ...Nosten, F.H. (2012). Adverse effects of falciparum and vivax malaria and the safety of antimalarial treatment in early pregnancy: a population-based study. Lancet Infect Dis, 12: 388-396.

[20]. McGready R., White N., Nosten F. (2011). Parasitological efficacy of antimalarials in the treatment and prevention of falciparum malaria in pregnancy 1998 to 2009: a systematic review. BJOG;118:123-135

[21]. Mutulei, A.C.N. (2013).Factors Influencing the Uptake of Intermittent Preventive Treatment for Malaria in Pregnancy: Evidence from Bungoma East District, Kenya. American Journal of Public Health Research, 1(5), 110-123 Available from http://pubs.sciepub.com/ajphr/1/5/2. Accessed June 262017.

[22]. National Malaria Elimination Programme (NMEP), National Population Commission (NPoPC), National Bureau of Statistics (NBS), \& ICF International. 2016). Nigeria Malaria Indicator Survey 2015: Key Indicators. Abuja, Nigeria, and Rockville, Maryland, USA: NMEP, NPoPC, and ICF International.

[23]. Nayak S., Varambally, K.V.M. (2017). Impact of Autonomy on Health-seeking Behaviour: Evidence from Rural India. Journal of Health Management 19(1) 109-120 DOI: 10.1177/0972063416682889

[24]. Nduka FO, Nwosu E, Oguariri RM. (2011). Evaluation of the effectiveness and compliance of IPTp in the control of malaria in pregnant women in southeastern Nigeria. Ann Trop Med Parasitol, 105:599-605.

[25]. Nganda, R.Y., Drakeley, C., Reyburn, H., \& Marchant, T. (2004). Knowledge of malaria influences the use of insecticide treated nets but not intermittent presumptive treatment by pregnant women in Tanzania. Malaria Journal, 3:42 doi: 10.1186/1475-2875-3-42. Available from: http://www.malariajournal.com/content/3/1/42. Accessed June 202015.

[26]. Olukemi, T.O., Emmanuel, O.A., \& Olusina, A.O. (2009).The use of intermittent preventive treatment with sulphadoxine-pyrimethamine in pregnancy in Ibadan, Nigeria: Implications for policy. Journal of Public Health and Epidemiology 1(1) 001-006.

[27]. Onoka, C.A., Hanson, K., Onwujekwe, O. E. (2012). Low coverage of intermittent preventive treatment for malaria in pregnancy in Nigeria: demand-side influences. . Malaria Journal, 11:82. Available from http://www.malariajournal.com/content/11/1/82. Accessed September 142016.

[28]. Owusu-Boateng, I., \& Anto, F. (2017). Intermittent preventive treatment of malaria in pregnancy: a cross-sectional survey to assess uptake of the new sulfadoxine-pyrimethamine five dose policy in Ghana. Malar J 16:323 DOI 10.1186/s12936-017-1969-7.

[29]. Peter, A.O. (2013). Effect of intermittent preventive treatment of malaria on the outcome of pregnancy among women attending antenatal clinic of a new Nigerian teaching hospital, Ado-Ekiti. Niger Med J, 54(3): 170-175. doi: 10.4103/0300-1652.114582.

[30]. Russell, T.L., Beebe, N.W., Cooper, R.D., Lobo, N.F., \& Burkot, T.R. (2013). Successful malaria elimination strategies require interventions that target changing vector behaviours. Malaria Journal, 12:56. Available from http://www.malariajournal.com/content/12/1/56. Accessed August 122017.

[31]. Shulman, C.E., Graham, W.J., Jilo, H., Lowe, B.S., New, L., Obiero, J., .. Marsh, K. (1996). Malaria as an important cause of anemia in primigravidae: evidence from a district hospital in coastal Kenya. Trans $R$ Soc Trop Med Hyg, 90:535-539.

[32]. Sikambale, C., Halwindi, H., \& Baboo, K.S. (2013). Factors Influencing Utilization of Intermittent Presumptive Treatment of Malaria (IPTp) Services by Pregnant Women in Sesheke District of Western Province Zambia. Medical Journal of Zambia, 40 (1) 24-32.

[33]. Sokhna, C., Ndiath, M.O., \& Rogier, C. (2013). The changes in mosquito vector behaviour and the emerging resistance to insecticides will challenge the decline of malaria. Clin Microbiol Infect; 19: 902-907. 
DOI: 10.21522/TIJPH.2013.06.01.Art009

ISSN: $2520-3134$

[34]. Steketee, R.W., Wirima, J.J., \& Campbell, C.C., (1996). Developing effective strategies for malaria prevention programmes for pregnant African women. American journal of tropical medicine and hygiene, 55 (1 Suppl): 95-100.

[35]. Takem, E.N., Achidi, E.A., Ndumbe, P.M. (2009). Use of intermittent preventive treatment for malaria by pregnant women in Beua, Cameroon. Acta Trop, 112:54-58.

[36]. Thomsen, E.K., Koimbu, G., Pulford, J., Jamea-Maiasa, S., Ura, Y., Keven, J.B., .. Reimer, L.J. (2017).Mosquito Behavior Change After Distribution of Bed nets Results in Decreased Protection Against Malaria Exposure. The Journal of Infectious Diseases, 215:790-7.

[37]. Verhoeff, F.H., Brabin, B.J., Chimsuku, L., Kazembe, P., Russel, W.B., \& Broadhead, R.L. (1998). An evaluation of intermittent sulfadoxine-pyrimethamine treatment in pregnancy on parasite clearance and risk of low birth weight in rural Malawi. Ann Trop Med Parasitol, 92:141-150.

[38]. Woldemicael, G. (2007). Do women with higher autonomy seek more maternal and child health-care? Evidence from Ethiopia and Eritrea. Available from http://www.demogr.mpg.de/papers/working/wp-2007035.pdf. Accessed June 262017.

[39]. World Health Organization (2011). WHO Global Malaria Programme: World Malaria Report. World Health Organization, Geneva, Switzerland.

[40]. World Health Organization. (2014). WHO policy brief for the implementation of intermittent preventive treatment of malaria in pregnancy using sulfadoxine-pyrimethamine (IPTp-SP) April 2013 (rev. January 2014).

[41]. World Health Organization. (2017). Malaria in pregnant women. Available from http://www.who.int/malaria/areas/high_risk_groups/pregnancy/en/. Accessed November 102017.

[42]. World Health Organization \&United Nations International Children Education Fund. (2003). Malaria in pregnancy. World Health Organization/UNICEF. WHO/CDS/MAL/2003. 1093. 